\title{
TEMPERATURA DE ARMAZENAMENTO E TIPO DE CORTE PARA MELÃO MINIMAMENTE PROCESSADO ${ }^{1}$
}

\author{
MARIA CECÍLIA DE ARRUDA², ANGELO PEDRO JACOMINO ${ }^{3}$, RICARDO ALFREDO KLUGE ${ }^{4}$, MARISA AZZOLINI ${ }^{5}$
}

\begin{abstract}
RESUMO - O presente trabalho teve como objetivo determinar a temperatura de armazenamento e o tipo de corte que proporciona melhor manutenção da qualidade de melões minimamente processados. Melões rendilhados, híbrido Bonus II, foram processados em câmara fria a $12^{\circ} \mathrm{C}$. Os frutos foram cortados manualmente em 8 fatias longitudinais. Em um dos tratamentos, as fatias foram divididas em pedaços de aproximadamente $3 \mathrm{~cm}$ de base e, no outro tratamento, foram utilizadas fatias inteiras. O produto minimamente processado foi acondicionado em embalagem rígida de politereftalato de etileno e armazenado a $3 ; 6$ e $9^{\circ} \mathrm{C}$. O delineamento experimental foi inteiramente casualizado em esquema fatorial. Foram realizadas análises físicoquímicas e sensoriais a cada 3 dias, por um período de 9 dias. A coloração e o teor de sólidos solúveis totais não foram afetados pelos tratamentos. $\mathrm{O}$ produto armazenado a $3^{\circ} \mathrm{C}$ manteve maiores valores de firmeza, independentemente do tipo de corte. A aparência foi considerada boa até o $9^{\circ}$ dia de armazenamento e o aroma, até o $6^{\circ}$ dia, para melões a $3^{\circ} \mathrm{C}$. Em todos os tratamentos, houve declínio das notas atribuídas ao sabor durante o armazenamento. Pelos resultados obtidos, conclui-se que a qualidade de melões minimamente processados pode ser mantida por 6 dias a $3^{\circ} \mathrm{C}$, independentemente do tipo de corte.
\end{abstract}

Termos para indexação: Cucumis melo L., processamento, armazenamento refrigerado, qualidade

\section{STORAGE TEMPERATURE AND CUT TYPE TO MINIMALLY PROCESSED MELON}

ABSTRACT - The objective of this work was to determine the storage temperature and the cut type that provides the better maintenance of the quality of minimally processed melons (Cucumis melo L. var. reticulatus hybrid. Bonus II). Fruits were hand cut in 8 longitudinal slices in cold chamber at $12^{\circ} \mathrm{C}$. One of the slices was divided in $3 \mathrm{~cm}$ pieces, in the other treatment whole slices were used. The product minimally processed was packed in rigid polyethylene terephthalate tray and stored at 3,6 and $9^{\circ} \mathrm{C}$. The experimental design was completely randomized in factorial arrangement. The physicalchemical and sensorial characteristics were analyzed every 3 days during 9 days. The coloration and total soluble solids were not affected by the treatments. The product stored at $3^{\circ} \mathrm{C}$ had higher efficiency maintaining firmness independently of the cut type. The appearance was considered good until $9^{\text {th }}$ storage day and the aroma until $6^{\text {th }}$ storage day, for melons at $3^{\circ} \mathrm{C}$. The flavor declined during the storage in all treatments. Minimally processed melons can be maintained until 6 days at $3^{\circ} \mathrm{C}$, independently of the cut type.

Index terms: Cucumis melo L., processing, cold storage, quality

\section{INTRODUÇÃO}

O processamento mínimo inclui operações de seleção, lavagem, corte, sanitização, centrifugação, embalagem, armazenamento e comercialização, de modo a obter um produto comestível fresco, saudável e que não necessite de subseqüente preparo (Moretti, 1999).

Nos EUA, a venda de produtos minimamente processados alcançou US\$ 7,9 bilhões em 1997, prevendo-se crescimento para US\$ 19 bilhões até 2003. No Brasil, o setor movimentou R \$ 450 milhões em 1998 (FNP, 2000). É uma atividade que está em expansão, devido à necessidade que os consumidores têm de adquirir produtos com qualidade e praticidade (Wiley, 1994).

Hortaliças e frutos minimamente processados são mais perecíveis do que quando intactos, porque são submetidos a severo estresse físico advindo principalmente do descascamento e corte. $\mathrm{O}$ efeito do corte leva a um aumento da taxa respiratória e produção de etileno, com aumento da atividade enzimática devido à ruptura de muitas células (Chitarra, 1998).

De acordo com Brecht (1995), quanto maior a gravidade da injúria nos tecidos, maior é a velocidade de deterioração dos produtos minimamente processados. Assim, é de esperar-se que diferentes tipos de corte promovam diferentes respostas quanto à qualidade dos produtos minimamente processados.

As mudanças bioquímicas em frutos e vegetais minimamente processados, são, em parte, conseqüência do efeito da temperatura na atividade enzimática. Em temperaturas superiores a $10^{\circ} \mathrm{C}$, a concentração de $\mathrm{CO}_{2}$ aumenta abruptamente devido à intensificação do metabolismo e proliferação microbiana (Varoquaux \& Wiley, 1994).

O controle da temperatura é uma das técnicas mais usuais e importantes para minimizar o efeito do corte em frutos e hortaliças (Brecht,
1995). Allong et al. (2000) verificaram que a temperatura de $5^{\circ} \mathrm{C}$ foi mais eficiente que $10^{\circ} \mathrm{C}$ em retardar o crescimento microbiano e preservar a qualidade sensorial de mangas minimamente processadas. $\mathrm{O}$ objetivo deste trabalho foi determinar a temperatura de armazenamento e tipo de corte que proporcionem melhor manutenção da qualidade de melões minimamente processados.

\section{MATERIALEMÉTODOS}

Melões rendilhados híbridos. Bônus II provenientes do Rio Grande do Norte foram obtidos na Ceasa-Campinas e levados ao Laboratório de Pós-Colheita do Departamento de Produção Vegetal da Esalq/ USP, onde foram selecionados quanto à ausência de danos mecânicos e grau de maturação. Utilizaram-se frutos com aproximadamente $1,2 \mathrm{~kg}$. Estes foram lavados com detergente, desinfetados com hipoclorito de sódio (100 ppm) e armazenados a $10^{\circ} \mathrm{C}$, por $12 \mathrm{~h}$.

Decorrido o tempo de armazenamento, os frutos foram cortados ao meio e suas sementes eliminadas com auxílio de uma colher. Cada metade foi cortada em 4 fatias longitudinais e, em seguida, as cascas foram eliminadas. Em um dos tratamentos, as fatias foram divididas em pedaços menores com aproximadamente $3 \mathrm{~cm}$ de aresta (cubos) e, no outro, foram utilizadas as fatias inteiras. Os cubos e as fatias foram imersos em água clorada (100 ppm) por 3 segundos e drenados em escorredor doméstico. $\mathrm{O}$ acondicionamento foi feito em embalagem rígida de politereftalato de etileno, com capacidade para $750 \mathrm{~mL}$. Todos estes procedimentos foram realizados em câmara fria a $12^{\circ} \mathrm{C}$ sob condições higiênicas. $\mathrm{O}$ armazenamento foi realizado a $3^{\circ} \mathrm{C}, 6^{\circ} \mathrm{C}$ e $9^{\circ} \mathrm{C}$, durante 9 dias.

O delineamento experimental utilizado foi o inteiramente ao acaso, em esquema fatorial $2 \times 3 \times 4$ ( 2 tipos de corte, 3 temperaturas de

\footnotetext{
1 (Trabalho 089/2002). Recebido: 14/06/2002; Aceito para publicação:13/02/2003. Apoio FAPESP.

${ }^{2}$ Mestranda em Fitotecnia - ESALQ/USP, CEP: 13418-900, Piracicaba-SP, mcarruda@esalq.usp.br, (19) 3429-4190.

${ }^{3}$ Prof. Dr. - Departamento de Produção Vegetal, ESALQ/USP, jacomino@ esalq.usp.br, (19) 3429-4190.

${ }^{4}$ Prof. Dr. - Departamento de Ciências Biológicas, ESALQ/USP, rakluge@ esalq.usp.br (19) 3429-4160.

${ }^{5}$ Mestranda em Fisiologia e Bioquímica de Plantas - ESALQ/USP, mazzolin@esalq.usp.br (19) 3429-4160.
} 
armazenamento e 4 períodos de avaliação). Utilizaram-se 5 repetições, sendo cada uma representada por uma bandeja contendo aproximadamente $240 \mathrm{~g}$ do produto minimamente processado.

Foram realizados testes de aceitabilidade do produto e análises físico-químicas a cada 3 dias, por um período de 9 dias. As variáveis físico-químicas analisadas foram: a) escurecimento: determinado com colorímetro Minolta, modelo CR-300, tomando-se leituras na região placentária. As leituras foram realizadas em 6 cubos e duas fatias por repetição e os resultados expressos em Luminosidade $\left(\mathrm{L}^{*}\right)$; b) firmeza da polpa: determinada com penetrômetro digital, ponteira $8 \mathrm{~mm}$, tomandose uma leitura na região placentária do cubo e da fatia. As leituras foram realizadas em 6 cubos e em 2 fatias, sendo os resultados expressos em N; c) teor de sólidos solúveis totais (SST): leitura direta em refratômetro digital Atago modelo Palete 101, utilizando-se de polpa homogeneizada em triturador doméstico tipo 'mixer'. Os resultados foram expressos em ${ }^{\circ}$ Brix. O teste de aceitabiliade foi realizado por uma equipe de 5 provadores, os quais atribuíram notas de aparência, aroma e sabor, segundo a escala descrita a seguir:

Aparência: 5=ótimo (melão com aspecto de frescor, ausência de escurecimento, translucência e bolores); 4 =bom (melão com aspecto de frescor, porém com leve escurecimento na região placentária e/ou leve translucência e ausência de bolores); $\mathbf{3}=$ regular (melão com pouco aspecto de frescor, moderado escurecimento na região placentária e/ou moderada translucência e ausência de bolores); 2 =ruim (melão sem aspecto de frescor, elevado escurecimento na região placentária e/ou elevado grau de translucência e ausência de bolores); 1=péssimo (melão com elevado grau de translucência e bolores).

Aroma: 5=ótimo (melão bastante aromático - típico de net melon); 4=bom (melão com aroma moderado); $3=$ =regular (melão com aroma fraco ou sem aroma); 2=ruim (melão com aroma alcoólico); $1=$ péssimo (melão com aroma de produto putrefato).

Sabor: 5=ótimo (sabor característico); 4= bom (leve perda do sabor característico); $3=$ regular (ausência de sabor característico); $2=$ ruim (sabor levemente alcoólico); 1=péssimo (sabor alcoólico).

A nota 3 foi considerada limite de aceitabilidade. Os pedaços de melões foram mantidos por 3 horas sob temperatura ambiente, após serem retirados das câmaras frias e, em seguida, oferecidos aos provadores. Os resultados foram submetidos à análise de variância pelo teste $\mathrm{F}$ e comparação de médias pelo teste Tukey (5\%).

\section{RESULTADOSEDISCUSSÃO}

Observa-se pela Tabela 1 que houve redução nos valores de Luminosidade (L) nas três temperaturas de armazenamento, sendo a mudança de coloração significativa apenas no decorrer do período de armazenamento, ou seja, não houve efeito de corte nem temperatura. A redução da luminosidade durante o período de armazenamento também foi relatada por Pinto (2002) em melão ‘Orange Flesh' minimamente processado, armazenado a $3^{\circ} \mathrm{C}$. A variável luminosidade diferencia cores claras de escuras. Seu valor varia de zero para cores escuras a 100 para cores claras. Quanto menor o valor desta variável, maior o grau de depreciação da aparência do produto.

TABELA 1- Escurecimento da polpa do melão rendilhado minimamente processado durante $\mathrm{o}$ armazenamento refrigerado ${ }^{1,2)}$

\begin{tabular}{ccccc}
\hline Temperaturas de & \multicolumn{4}{c}{ Dias de armazenamento } \\
\cline { 2 - 5 } Armazenamento & $\mathbf{0}$ & $\mathbf{3}$ & $\mathbf{6}$ & $\mathbf{9}$ \\
\hline & $------------~$ & Luminosidade (L) ---------- \\
3C & $49,12 \mathrm{~A}$ & $46,11 \mathrm{AB}$ & $47,96 \mathrm{~A}$ & $41,76 \mathrm{~B}$ \\
6C & $49,12 \mathrm{~A}$ & $44,71 \mathrm{AB}$ & $45,40 \mathrm{AB}$ & $42,48 \mathrm{~B}$ \\
9C & $49,12 \mathrm{~A}$ & $46,45 \mathrm{~A}$ & $44,22 \mathrm{AB}$ & $40,53 \mathrm{~B}$ \\
\hline Médias & $49,12 \mathrm{~A}$ & $45,76 \mathrm{~B}$ & $45,86 \mathrm{~B}$ & $41,59 \mathrm{C}$ \\
\hline
\end{tabular}

CV (\%): 10,31

${ }^{1)}$ Médias seguidas de mesma letra maiúscula na linha não diferem entre si, pelo teste de Tukey, ao nível de $5 \%$ de probabilidade.

2) Os valores são referentes à média de coloração obtida entre os dois tipos de corte.
A mudança de coloração durante o período de armazenamento caracterizou-se por um leve escurecimento na região placentária. Em frutas e hortaliças minimamente processadas, existem vários tipos de reações oxidativas, as quais causam escurecimento do tecido (Wiley, 1994). O uso de aditivos para a preservação da coloração de produtos minimamente processados tem sido alvo de estudo para muitos pesquisadores. Lamikanra \& Watson (2000) verificaram que a utilização de ácido ascórbico preservou a coloração de melão Cantaloupe minimamente processado por 25 dias, a $4^{\circ} \mathrm{C}$.

Verifica-se, na Tabela 2, uma redução significativa da firmeza da polpa de melão minimamente processado, com o decorrer do tempo e do aumento da temperatura de armazenamento. Ao final de 9 dias de armazenamento, a firmeza da polpa dos melões correspondia a 79,3\%, $54,3 \%$ e 49,2\% da firmeza inicial, para o produto armazenado a $3 ; 6$ e $9^{\circ} \mathrm{C}$, respectivamente. Portela \& Cantwell (1998) verificaram um decréscimo de $28 \%$ da firmeza em pedaços de melão Cantaloupe armazenados a $5^{\circ} \mathrm{C}$, por 12 dias.

TABELA 2- Firmeza da polpa de melão rendilhado minimamente processado durante o armazenamento refrigerado ${ }^{1,2)}$

\begin{tabular}{|c|c|c|c|c|c|}
\hline \multirow{2}{*}{$\begin{array}{l}\text { Temperaturas de } \\
\text { Armazenamento }\end{array}$} & \multicolumn{5}{|c|}{ Dias de armazenamento } \\
\hline & 0 & 3 & 6 & 9 & Médias \\
\hline & & (5) & Tewton $(\mathrm{N})$ & & \\
\hline $3 \mathrm{C}$ & $4,46 \mathrm{a} A$ & $4,22 \mathrm{a} A B$ & $4,03 \mathrm{aAB}$ & $3,49 a \mathrm{~B}$ & $4,05 \mathrm{a}$ \\
\hline $6 \mathrm{C}$ & $4,46 \mathrm{a} A$ & $3,70 \mathrm{a} A$ & $3,52 \mathrm{a} \mathrm{A}$ & $2,42 \mathrm{~b} \mathrm{~B}$ & $3,52 \mathrm{~b}$ \\
\hline $9 \mathrm{C}$ & $4,46 \mathrm{a} A$ & $3,37 \mathrm{a} \mathrm{B}$ & $3,30 \mathrm{a} B$ & $2,19 \mathrm{~b} \mathrm{C}$ & $3,33 \mathrm{~b}$ \\
\hline Médias & $4,46 \mathrm{~A}$ & $3,76 \mathrm{~B}$ & $3,61 \mathrm{~B}$ & $2,70 \mathrm{C}$ & \\
\hline
\end{tabular}

$\mathrm{CV}(\%): 22,71$

${ }^{1)}$ Médias seguidas de mesma letra minúscula na coluna e maiúscula na linha não diferem entre si, pelo teste de Tukey, ao nível de 5\% de probabilidade.

2) Os valores são referentes à média de firmeza obtida entre os dois tipos de corte.

Verifica-se também, na Tabela 2, que a firmeza da polpa dos melões armazenados a $3^{\circ} \mathrm{C}$ foi significativamente superior àqueles armazenados nas demais temperaturas. Portela et al. (1997) citam a temperatura como fator mais importante para conservação de melão minimamente processado. Estes autores, observaram que pedaços de melões mantidos a $5^{\circ} \mathrm{C}$, por 9 dias, apresentaram maior firmeza do que a $10^{\circ} \mathrm{C}$.

Os teores de sólidos solúveis totais apresentaram interação significativa $(\mathrm{P}<0,05)$ entre os tipos de corte e o período de armazenamento. De maneira geral, houve acréscimo no teor de sólidos solúveis totais durante o armazenamento (Tabela 3). Estes dados são discordantes daqueles obtidos por Shellie \& Saltveit (1993), os quais verificaram, em melões rendilhados, comportamento constante do teor de sólidos solúveis totais durante todo período de armazenamento.

TABELA 3- Teores de sólidos solúveis totais (SST) de melão rendilhado minimamente processado durante o armazenamento refrigerado $^{1,2)}$

\begin{tabular}{|c|c|c|c|c|c|}
\hline \multirow{2}{*}{$\begin{array}{l}\text { Tipos de } \\
\text { corte }\end{array}$} & \multicolumn{5}{|c|}{ Dias de armazenamento } \\
\hline & 0 & 3 & 6 & 9 & Médias \\
\hline & & -SST $\left({ }^{\circ} \mathrm{Br}\right.$ & x) ----------- & & $\cdots$ \\
\hline Fatia & $11,64 a$ B & $12,10 \mathrm{aAB}$ & $12,45 \mathrm{aAB}$ & $13,10 \mathrm{aA}$ & $12,32 \mathrm{a}$ \\
\hline Cubo & $11,64 \mathrm{a} A B$ & $12,24 \mathrm{a} A$ & $11,20 \mathrm{~b} \mathrm{~B}$ & $12,11 \mathrm{bA}$ & $11,80 \mathrm{~b}$ \\
\hline Médias & $11,64 \mathrm{~A}$ & $12,17 \mathrm{AB}$ & $11,83 \mathrm{~B}$ & $12,70 \mathrm{~A}$ & \\
\hline
\end{tabular}

${ }^{1)}$ Médias seguidas de mesma letra minúscula na coluna e maiúscula na linha não diferem entre si, pelo teste de Tukey, ao nível de 5\% de probabilidade.

2) Os valores são referentes à média de sólidos solúveis totais nas três temperaturas de armazenamento.

Os melões fatiados apresentaram, a partir do $6^{\circ}$ dia de armazenamento, valores significativamente maiores de sólidos solúveis totais em relação aos melões cortados na forma de cubos. Isto pode ser 
devido a um menor metabolismo dos melões cortados em fatias, o que levou a um menor consumo de reservas.

Observa-se, na Tabela 4, que, ao final do período de armazenamento, os melões mantidos a $3^{\circ} \mathrm{C}$ apresentaram notas significativamente maiores de aparência, em relação aos melões mantidos nas demais temperaturas. Verifica-se também que não houve alteração significativa desta variável, para os melões armazenados a $3^{\circ} \mathrm{C}$. O'ConnorShaw et al. (1994) também não observaram mudanças significativas na aparência de melões honeydew minimamente processados armazenados a $4^{\circ} \mathrm{C}$, por 14 dias.

TABELA 4- Aparência de melão rendilhado minimamente processado durante o armazenamento refrigerado (Notas: 5=ótimo; 4=bom; 3=regular; 2=ruim; $1=$ péssimo) ${ }^{1,2)}$

\begin{tabular}{cccccc}
\hline Temperaturas de & \multicolumn{5}{c}{ Dias de armazenamento } \\
\cline { 2 - 6 } Armazenamento & 0 & 3 & 6 & 9 & Médias \\
\hline 3C & $5,0 \mathrm{a} A$ & $3,8 \mathrm{a} \mathrm{A}$ & $4,2 \mathrm{a} A$ & $3,9 \mathrm{a} A$ & $4,23 \mathrm{a}$ \\
$6 \mathrm{C}$ & $5,0 \mathrm{a} A$ & $4,0 \mathrm{a} A$ & $3,8 \mathrm{ab} \mathrm{A}$ & $2,4 \mathrm{~b} \mathrm{~B}$ & $3,80 \mathrm{a}$ \\
9C & $5,0 \mathrm{a} A$ & $3,4 \mathrm{a} \mathrm{B}$ & $2,6 \mathrm{~b} \mathrm{~B}$ & $1,0 \mathrm{c} \mathrm{C}$ & $3,00 \mathrm{~b}$ \\
\hline Médias & $5,00 \mathrm{~A}$ & $3,73 \mathrm{~B}$ & $3,53 \mathrm{~B}$ & $2,43 \mathrm{C}$ \\
\end{tabular}

CV (\%): 32,57

${ }^{1)}$ Médias seguidas de mesma letra minúscula na coluna e maiúscula na linha não diferem entre si, pelo teste de Tukey, ao nível de 5\% de probabilidade.

${ }^{2)}$ Os valores são referentes à média de aparência obtida entre os dois tipos de corte.

Os melões mantidos a $6^{\circ} \mathrm{C}$ apresentaram aspecto translúcido e notas de aparência abaixo do limite de aceitabilidade (menor que 3), ao final do armazenamento. Os melões armazenados a $9^{\circ} \mathrm{C}$ apresentaram-se impróprios para o consumo já aos 6 dias de armazenamento (nota de aparência inferior a 3), devido ao aspecto translúcido, e aos 9 dias apresentou desenvolvimento de bolores em forma de colônias brancas.

$\mathrm{O}$ aroma foi considerado bom a regular até o $6^{\circ}$ dia em todos os tratamentos (Tabela 5). No $9^{\circ}$ dia, verificou-se um decréscimo significativo na nota atribuída ao aroma dos melões mantidos a $9^{\circ} \mathrm{C}$, posicionandoo abaixo do limite de aceitabilidade.

TABELA 5- Aroma de melão rendilhado minimamente processado durante $\mathrm{o}$ armazenamento refrigerado.(Notas: $5=$ =ótimo; $4=$ =bom; $3=$ regular; $2=$ ruim; $1=$ péssimo $)^{1,2)}$

\begin{tabular}{|c|c|c|c|c|c|}
\hline \multirow{2}{*}{$\begin{array}{l}\text { Temperaturas de } \\
\text { Armazenamento }\end{array}$} & \multicolumn{5}{|c|}{ Dias de armazenamento } \\
\hline & 0 & 3 & 6 & 9 & Médias \\
\hline $3 \mathrm{C}$ & $5,0 \mathrm{a} A$ & $4,0 \mathrm{a} \mathrm{AB}$ & $4,0 \mathrm{a} \mathrm{AB}$ & $3,5 \mathrm{a} \mathrm{B}$ & $4,13 \mathrm{a}$ \\
\hline $6 \mathrm{C}$ & $5,0 \mathrm{a} \mathrm{A}$ & $3,9 \mathrm{a} A B$ & $3,5 \mathrm{a} \mathrm{B}$ & $3,3 \mathrm{a} \mathrm{B}$ & $3,93 \mathrm{ab}$ \\
\hline $9 \mathrm{C}$ & $5,0 \mathrm{a} A$ & $3,8 \mathrm{a} A B$ & $3,0 \mathrm{a} B$ & $1,8 \mathrm{~b} \mathrm{C}$ & $3,40 \mathrm{~b}$ \\
\hline Médias & $5,00 \mathrm{~A}$ & $3,90 \mathrm{~B}$ & $3,50 \mathrm{BC}$ & $2,87 \mathrm{C}$ & \\
\hline
\end{tabular}

Em todos os tratamentos, houve declínio das notas atribuídas ao sabor durante o armazenamento, sendo que, no $6^{\circ}$ dia, os melões mantidos a $3^{\circ} \mathrm{C} \mathrm{e} 6^{\circ} \mathrm{C}$ apresentaram sabor aceitável, enquanto aqueles armazenados a $9^{\circ} \mathrm{C}$ apresentavam sabor ruim (Tabela 6). No $9^{\circ}$ dia, não se efetuou avaliação do sabor, devido à presença de bolores nos melões armazenados a $9^{\circ} \mathrm{C}$.

TABELA 6. Sabor de melão rendilhado minimamente processado durante o armazenamento refrigerado.(Notas: 5=ótimo; 4=bom; $3=$ regular; 2=ruim; 1=péssimo) ${ }^{1,2)}$

\begin{tabular}{ccccc}
\hline Temperatura de & \multicolumn{4}{c}{ Dias de armazenamento } \\
\cline { 2 - 5 } Armazenamento & $\mathbf{0}$ & $\mathbf{3}$ & $\mathbf{6}$ & Médias \\
\hline 3C & $5,0 \mathrm{a} \mathrm{A}$ & $3,9 \mathrm{a} \mathrm{AB}$ & $3,2 \mathrm{a} \mathrm{B}$ & $4,03 \mathrm{a}$ \\
6C & $5,0 \mathrm{a} \mathrm{A}$ & $3,1 \mathrm{a} \mathrm{B}$ & $3,0 \mathrm{a} \mathrm{B}$ & $3,70 \mathrm{a}$ \\
9C & $5,0 \mathrm{a} \mathrm{A}$ & $3,7 \mathrm{a} \mathrm{B}$ & $2,2 \mathrm{a} \mathrm{C}$ & $3,63 \mathrm{a}$ \\
\hline Médias & $5,00 \mathrm{~A}$ & $3,57 \mathrm{~B}$ & $2,80 \mathrm{C}$ \\
\hline
\end{tabular}

CV (\%): 30,51 diferem entre si, pelo teste de Tukey, ao nível de $5 \%$ de probabilidade.

${ }^{2)}$ Os valores se referem à média de sabor obtida entre os dois tipos de corte.
Pelo presente trabalho, verificou-se que ocorreu um escurecimento da região placentária dos melões durante o armazenamento em todas as temperaturas. Os teores de sólidos solúveis totais variaram com o tipo de corte, sendo que os melões fatiados apresentaram consumo significativamente menor em relação aos melões em cubos. No entanto, essa diferença não alterou as características sensoriais do produto.

Os melões minimamente processados, armazenados a $3^{\circ} \mathrm{C}$, apresentaram melhor firmeza da polpa e melhores características sensoriais ao final do armazenamento.

\section{CONCLUSÕES}

Pelos resultados obtidos, conclui-se que:

1) A melhor temperatura para armazenamento do melão rendilhado, minimamente processado, é $3^{\circ} \mathrm{C}$.

2) Os tipos de cortes (fatia ou cubo) não influenciam a qualidade do melão rendilhado, minimamente processado.

3) O melão rendilhado, minimamente processado, mantém boa qualidade por até 6 dias a $3^{\circ} \mathrm{C}$

\section{REFERÊNCIASBIBLIOGRÁFICAS}

AGRIANUAL 2000: anuário da agricultura brasileira. São Paulo: FNP Consultoria \& Comércio, 2001, p.40

ALLONG, R.; WICKHAM, L.D.; MOHAMMED, M. The effect of cultivar, fruit ripeness, storage temperature and duration on quality of freshcut mango. Acta Horticulturae, Wageningen, n.509, p.487-494, 2000.

BRECHT, J.K. Physiology of lightly processed fruits and vegetables. HortScience, Alexandria, v.30, n.1, p.18-22, 1995.

CHITARRA, M.I.F. Processamento mínimo de frutos e hortaliças. Viçosa: Centro de Produções Técnicas, 1998. 88p.

LAMIKANRA, O.; WATSON, M.A. Cantaloupe melon peroxidase: Characterization and effects of additivies on activity. Nahrung, Weinheim, v.44, n.3, p.168-172, 2000.

MORETTI, C.L. Processamento mínimo de hortaliças: alternativa viável para a redução de perdas pós-colheita e agregação de valor ao agronegócio brasileiro. Horticultura Brasileira, Brasília, v.17, n.2, p.1, 1999.

O'CONNOR-SHAW, R.E.; ROBERTS, R.; FORD, A.L.; NOTTINGHAM, S.M. Shelf life of minimally processed honeydew, kiwifruit, papaya, pineapple and cantaloupe. Journal of Food Science, Chicago, v.59, n.6, p.1202-1206 e1215, 1994.

PORTELA, S.; NIE, X.; SUSLOW, T.; CANTWELL, M.; GORNY, J.R. Changes in sensory quality and fermentative volatile concentrations of minimally processed cantaloupe stored in controlled atmospheres. In: INTERNATIONALCONTROLLED RESEARCHCONFERENCE, 7., 1997, Davis. Proceedings. Davis: University of California, 1997. p.123-129.

PORTELA, S.I.; CANTWELL, M.I. Quality changes of minimally processed honeydew melons stored in air or controlled atmosphere. Postharvest Biology and Technology, Amsterdam, v.14, p.351-357, 1998.

SHELLIE, K.C.; SALTVEIT Junior., M.E. The lack of a respiratory rise in muskmelon fruit ripening on the plant challenges the definition of climacteric behaviour. Journal of Experimental Botany, Oxford, v.44, n.265, p.1403-1406, Aug.1993.

PINTO, S.A.A. Processamento mínimo de melão tipo Orange Flesh e de melanciaa 'Crimson Sweet'. 2002, 120f. Dissertação (Mestrado em Produção Vegetal) - Faculdade de Ciências Agrárias e Veterinárias, Universidade Estadual Paulista, Jaboticabal, 2002.

VAROQUAUX, P.; WILEY, R.C. Biological and biochemical changes in minimally processed refrigerated fruits and vegetables. In: WILEY, R.C. Minimally processed refrigerated fruits \& vegetables. New York: Chapman \& Hall, 1994. cap.6, p.226-268.

WILEY, R.C. Minimally processed refrigerated fruits and vegetables. New York: Chapman \& Hall, 1994. 368p. 